\title{
Ileoileal Intussusception Secondary to an lleal Fibroma
}

\author{
Haritha Chelimilla Ariyo Ihimoyan Simeon Carvajal \\ Balar Bhavna
}

Division of Gastroenterology, Bronx-Lebanon Hospital Center, Bronx, New York, N.Y., USA

\section{Key Words}

Ileoileal intussusception · Ileal fibroma · Intussusception · lleoileal · Fibroma

\begin{abstract}
Intussusception is defined as the telescoping of a segment of the gastrointestinal tract (intussusceptum) into an immediately adjacent distal bowel (intussuscipiens). Intussusception is a relatively rare cause of intestinal obstruction in adults. Unlike in children, a lead point is present in $90 \%$ of adult cases. The most common causes of small bowel intussusception are benign, usually hamartomas, lipomas, inflammatory polyps, adenomas and leiomyomas, in contrast to the large intestine where malignant tumors, usually adenocarcinomas, are more common. The clinical presentation of adult intussusception is non-specific with variable manifestations, predominantly those of intestinal obstruction, often making the diagnosis a challenge. The onset of symptoms may be acute, intermittent or chronic. We present a rare case of an ileal fibroma presenting with intussusception. A 43-year-old woman presented to our outpatient clinic with a history of recurrent abdominal pain. The clinical presentation and CT scan findings led to the diagnosis of ileoileal intussusception. Subsequently she underwent laparotomy which revealed an ileal fibroma as the lead point of the intussusception. Surgical exploration remains essential for diagnosis and treatment since in the majority of cases a pathologic lead point is identified. lleal fibroma is an uncommon benign neoplasm of the small bowel and must be considered in the differential diagnosis for small bowel intussusception.
\end{abstract}

\section{Introduction}

Intussusception is defined as the telescoping of a segment of the gastrointestinal tract (intussusceptum) into an immediately adjacent distal bowel (intussuscipiens). Intussusception was first reported by Paul Barbette of Amsterdam in in 1674 [1] and later by John Hunter in 1789 [2]. Intussusception accounts for only 1-5\% of all cases of intestinal obstruction in adults [3]. A lead point is a focal area of traction that can be 
either a mucosal, intramural or extraluminal lesion. A lead point is present in $90 \%$ of adult cases, unlike in children where it is most often idiopathic. Intussusceptions are classified according to their location in the gastrointestinal tract in order of frequency as enteric, colocolic, ileocecal, ileocolic, colorectal and rectorectal. In two thirds of cases a neoplasm is involved and nearly half are malignant [4]. The most common type of intussusception in adults is enteric, and the majority of the lesions (50-75\%) in this location are benign [4]. Secondary to non-specific presenting symptoms the diagnosis is often challenging and is usually confirmed at laparotomy. Surgery is the treatment of choice as in most cases the etiology involves an underlying pathologic process that acts as a lead point. Ileal fibroma is an uncommon benign neoplasm of the small bowel. We report a rare case of an ileal fibroma presenting with intussusception diagnosed by CT scan and confirmed by immunohistochemistry and present a brief review of the literature.

\section{Case Report}

A 43-year-old woman presented to our clinic with complaint of intermittent abdominal pain for the last 3 months. She had a history of occasional nausea with epigastric discomfort and mild constipation, but denied vomiting. There were no other associated symptoms. She denied any specific association with meals or defecation. She was seen in our gastroenterology clinic 2 months prior to this episode and at that time she had an upper gastrointestinal endoscopy to evaluate her symptoms, which revealed a hyperplastic polyp of the duodenal bulb about $3 \mathrm{~cm}$ in size and Helicobacter pylori gastritis which was treated subsequently. Her past medical history was significant for dyslipidemia and hypothyroidism. She denied any prior surgeries. Her home medications included atorvastatin, gemfibrozil and levothyroxine. She denied any significant family history of malignancies and any history of smoking or alcohol use or substance abuse. At the time of presentation to the clinic, her temperature was $97.9^{\circ} \mathrm{F}$, blood pressure $111 / 71 \mathrm{~mm} \mathrm{Hg}$, pulse 94 beats per minute, respiratory rate 20 breaths per minute and oxygen saturation $99 \%$ while she was breathing ambient air. At the time of presentation to the clinic, abdominal examination showed fullness in the epigastric region without any palpable mass and no hernia or scars. She was subsequently admitted for further evaluation of her pain due to worsening symptoms. Laboratory results were as follows: hemoglobin $9.5 \mathrm{~g} / \mathrm{dl}$, white blood cell count $1,200 / \mathrm{mm}^{3}$ (with $65 \%$ neutrophils and $24 \%$ lymphocytes), platelet count $254,000 / \mu \mathrm{l}$, prothrombin time $11.7 \mathrm{~s}$, activated partial thromboplastin time $25.6 \mathrm{~s}$, sodium $137 \mathrm{mEq} / \mathrm{l}$, potassium $3.8 \mathrm{mEq} / \mathrm{l}$, blood urea nitrogen $8 \mathrm{mg} / \mathrm{dl}$, creatinine $0.7 \mathrm{mg} / \mathrm{dl}$, total protein $7.3 \mathrm{~g} / \mathrm{dl}$, albumin $3.9 \mathrm{~g} / \mathrm{dl}$, alanine aminotransferase $23 \mathrm{U} / \mathrm{l}$, aspartate transaminase $20 \mathrm{U} / \mathrm{l}$, total bilirubin $0.2 \mathrm{mg} / \mathrm{dl}$, direct bilirubin $0.1 \mathrm{mg} / \mathrm{dl}$, serum lipase $37 \mathrm{U} / \mathrm{l}$ and serum ferritin $27.7 \mathrm{ng} / \mathrm{ml}$ (normal 30-400 ng/ml). Abdominal X-ray revealed proximal bowel obstruction with dilated bowel loops and air-fluid levels (fig. 1), and abdominal CT scan revealed ileoileal intussusception with a lead point which appeared to be a rounded solid mass in the right lower quadrant (fig. 2 ).

The patient underwent exploratory laparotomy with findings of ileoileal intussusception located about one foot from the ileocecal junction. When the ileoileal intussusception was reduced, a small round polyp, firm to hard in consistency, was found in the small bowel. Resection of the ileoileal intussusception with end-to-end anastomosis was performed with removal of few enlarged mesenteric lymph nodes in the adjacent mesentery. No other palpable tumor was found during exploration of the bowel. Postoperative recovery was uneventful. Histologically the polyp was involving the muscular wall of the bowel, consisting of fairly uniform-appearing round to oval cells with irregular nuclei with low mitotic activity and low Ki-67 index (fig. 3). There was admixture of lymphocytes, eosinophils, mast cells and plasma cells present in the lesion with delicate capillary vasculature. Immunohistochemical stains were negative for CD34, smooth muscle actin, S100, ALK-1, CD10, desmin, CD117 (KIT), DOG-1, pancytokeratin, EMA, CD45, CD20, CD3, CD68 (KPI and PGM), synaptophysin and chromogranin ( $\underline{\text { fig. } 4}$, fig. 5). Diffuse vimentin immunoreactivity of the tumor cells was noted (fig. 6). Histopathology was consistent with an ileal fibroma. The immunohistochemical stains helped distinguish the lesion from inflammatory fibroid polyp and gastrointestinal stromal tumor. Gastrointestinal stromal tumors typically express CD117 (KIT) and often CD34 while 
inflammatory fibroid polyps commonly express CD34 and S100 and are negative for c-kit, CD68, p53 and Bcl-2 stains [5]. Resected mesenteric lymph nodes showed reactive changes.

After an uneventful recovery, the patient was discharged on the fifth postoperative day. After 9 months, she is in good health.

\section{Discussion}

Intussusception is a rare cause of intestinal obstruction in adults. The most common type of intussusception in adults is enteric, where the underlying pathology is usually benign unlike in the colon. The most common lesions in small bowel intussusception are hamartomas, lipomas, inflammatory polyps, adenomas and leiomyomas [4], in contrast to the large intestine where malignant tumors, usually adenocarcinomas, are common. There are several case reports of ileoileal invagination in the literature, however intussusception secondary to fibromas is rare.

The presentation in adults is usually with vague symptoms such as abdominal pain, nausea and vomiting. Other sign and symptoms such as constipation, fever, intestinal bleeding, diarrhea and a palpable abdominal mass are less frequent. Preoperative diagnosis can be challenging due to its varying presentation. Onset of symptoms may be acute but may last for several weeks to a year before diagnosis [6]. Ileocolic intussusception due to an ileal polyp precipitated by colonoscopy has been reported [7]. Diagnostic workup with CT scan is useful with an accuracy of $80 \%[4,8]$. Since most patients present with obstructive symptoms, abdominal X-ray is most commonly performed as the initial test. Signs of intestinal obstruction such as dilated small bowel loops and air-fluid level may be seen, and information about the site of obstruction may be obtained. The characteristic features described on CT include an intraluminal soft tissue mass with an eccentrically placed fatty area or attenuation which may appear as 'target sign', 'sausage-shaped mass' or 'reniform mass' [9, 10]. CT scan and magnetic resonance imaging provide excellent preoperative evaluation in localizing the mass and define its relationship to the surrounding tissues. They are also useful for staging in the case of suspected malignancy and for assessing the presence of vascular compromise [11].

Surgery is the mainstay of treatment in most cases and is usually done emergently. There is no clear consensus about the optimal strategy for reduction before surgery. Our patient had reduction prior to resection for two reasons: firstly a shorter length of bowel involvement without any signs of ischemia and secondly the suspected benign etiology of the mass. Several factors need to be considered, such as the frequency of underlying pathology, possibility of malignancy, anatomical site, extent of the intussusception and presence of ischemia prior to consideration for reduction [12]. The overall incidence of malignancy in adults with intussusception is approximately $40 \%$, while for small bowel intussusception it is $40 \%$ compared to $65 \%$ in colonic intussusception [12-14]. In the study by Goh et al. [15], the site of intussusception in the colon and the presence of anemia were independent preoperative predictors of malignancy.

Fibroma is one of the rare benign tumors of the small intestine. More common benign neoplasms of the small intestine include adenomas, leiomyomas and lipomas. Immunohistochemistry pattern with positivity for vimentin and negative staining 
for CD34, smooth muscle actin, S100, ALK-1, CD10, desmin, CD117 (KIT), DOG-1, pancytokeratin, EMA, CD45, CD20, CD3, CD68 (KPI and PGM), synaptophysin and chromogranin confirmed the diagnosis. Malignant lesions causing intussusception in the small bowel are most often metastatic, melanoma being by far the most common cause of intussusception. Gastrointestinal stromal tumors and adenocarcinoma rarely cause ileal intussusceptions [16].

\section{Conclusion}

We report an unusual cause of small bowel obstruction in an adult patient, secondary to an ileal fibroma as the lead point of intussusception. Non-specific symptoms at presentation make early diagnosis difficult. Surgery remains the mainstay of management.
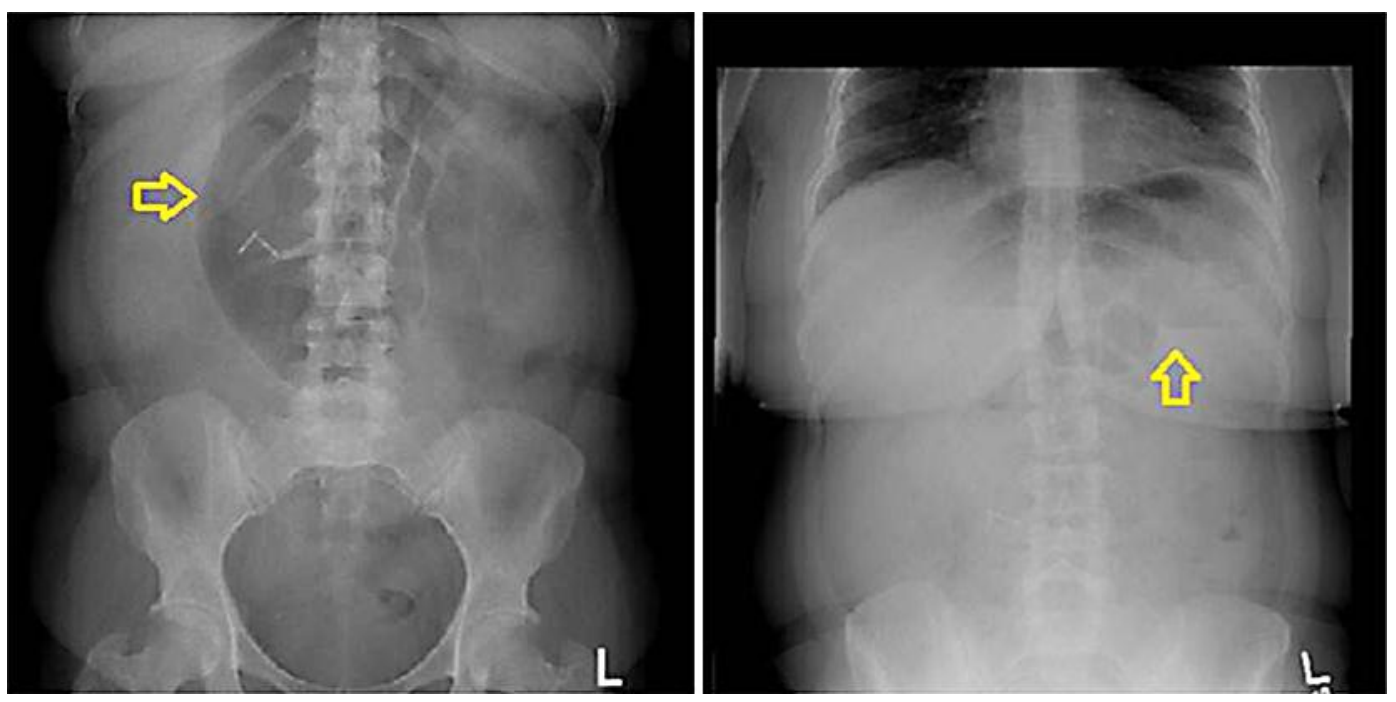

Fig. 1. Abdominal X-ray showing dilated proximal bowel loops with multiple air-fluid levels as indicated by arrows, suspicious of proximal bowel obstruction. 


\begin{tabular}{r|l|l|l}
$\begin{array}{r}\text { Case Reports in } \\
\text { Gastroenterology }\end{array}$ & $\begin{array}{l}\text { Case Rep Gastroenterol 2012;6:734-740 } \\
\text { DOI: 10.1159/000345806 }\end{array}$ & $\begin{array}{l}\text { Published online: } \\
\text { November 22, 2012 }\end{array}$ & $\begin{array}{l}\text { @ 2012 S. Karger AG, Basel } \\
\text { ISSN 1662-0631 } \\
\text { www.karger.com/crg }\end{array}$ \\
\hline
\end{tabular}

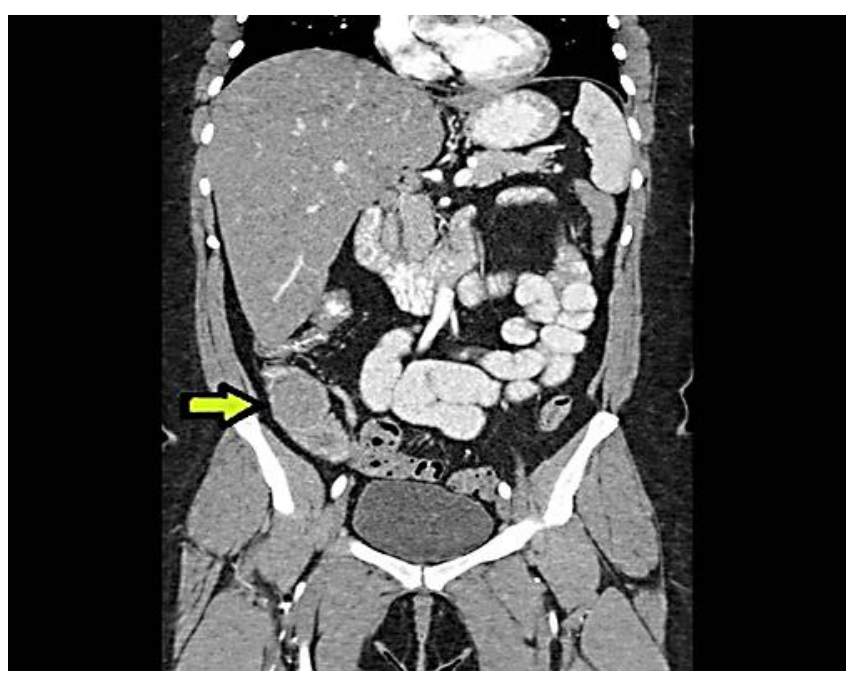

Fig. 2. Abdominal CT scan showing ileoileal intussusception with a lead point which appeared to be a rounded solid mass.

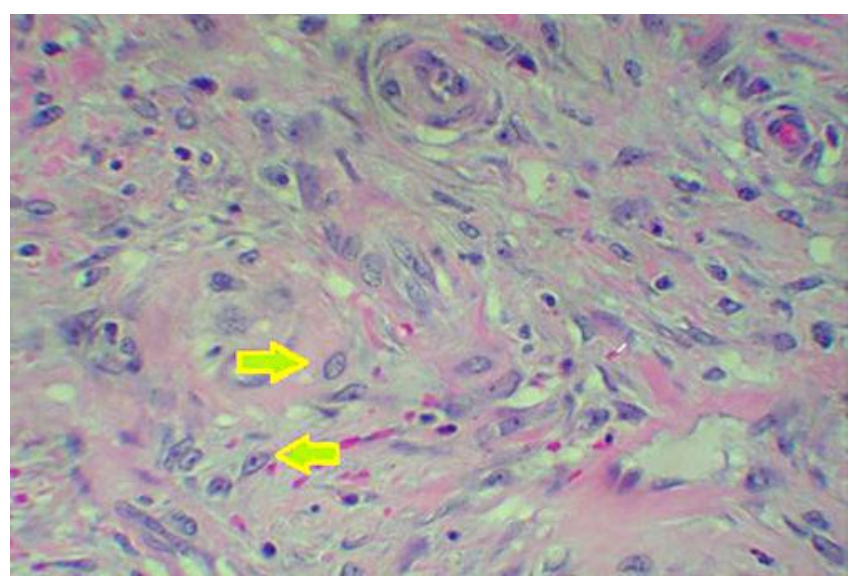

Fig. 3. H\&E stain of the ileal mass revealing fairly uniform-appearing round to oval cells with irregular nuclei containing delicate chromatin and several small distinct nucleoli. 

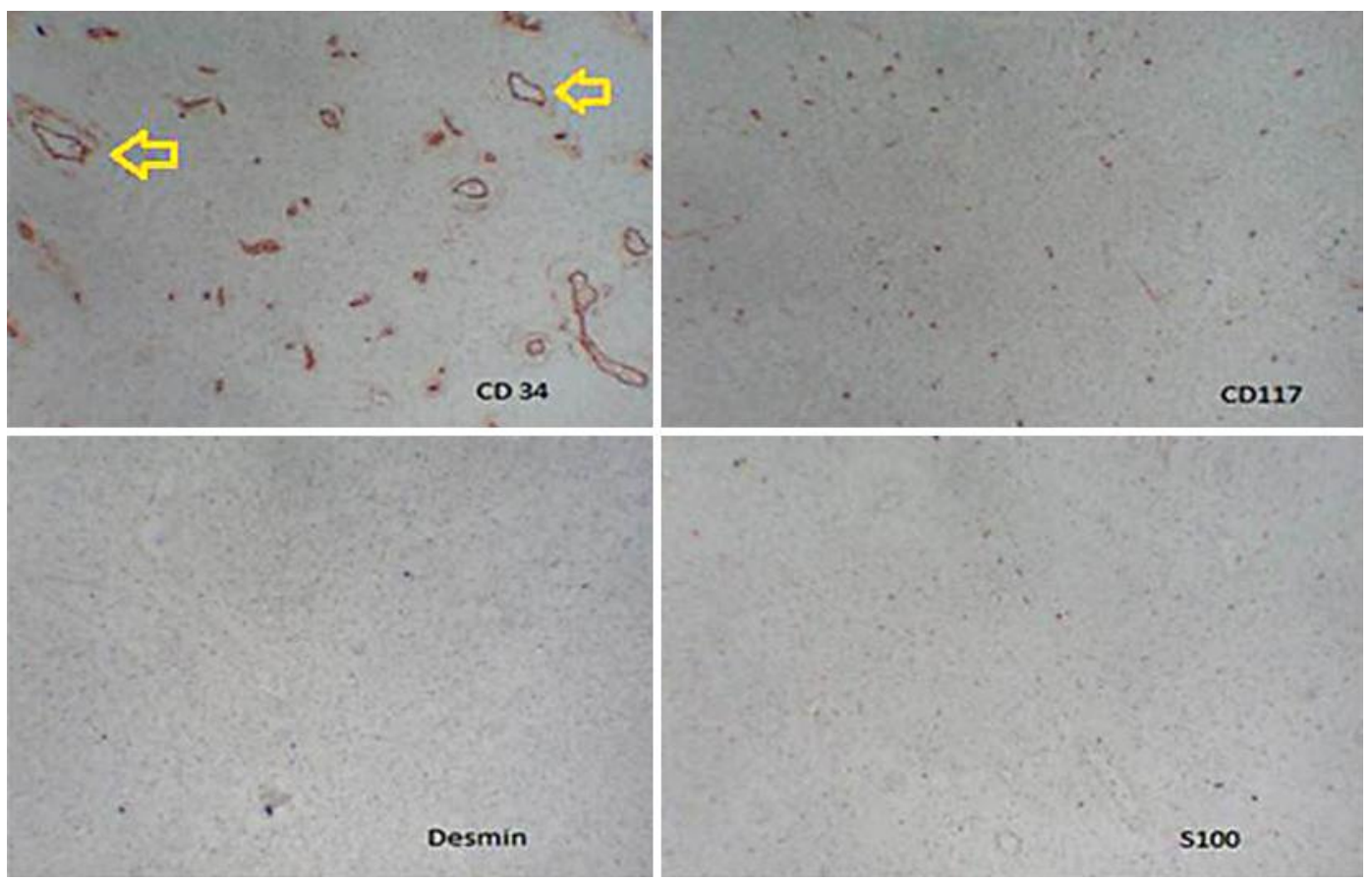

Fig. 4. Negative immunostaining for CD34 (the control being the vessel wall which is stained positive as indicated by the arrows), CD117, desmin and S100. Original magnification $\times 10$.

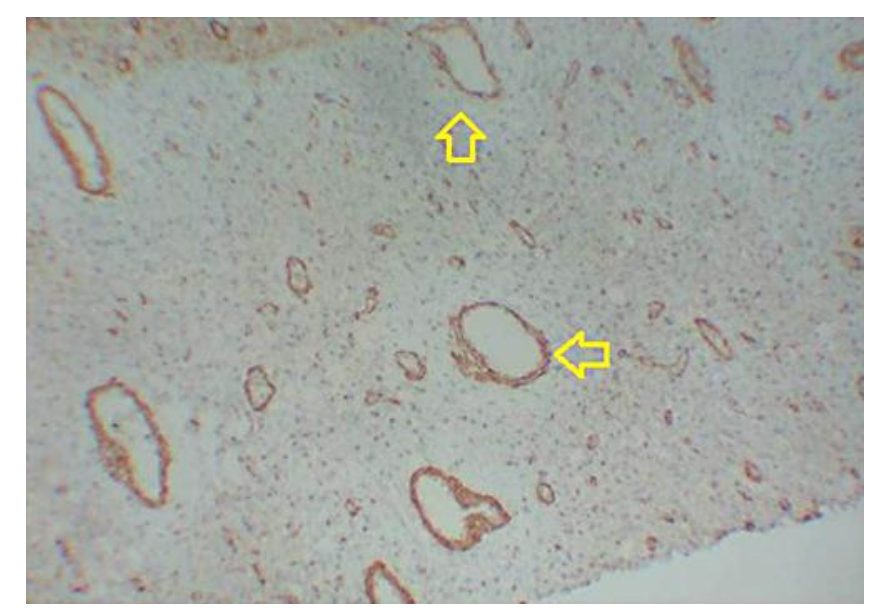

Fig. 5. Negative immunostaining for smooth muscle actin, with the control being the vessel wall which is stained positive as indicated by the arrows. Original magnification $\times 10$. 


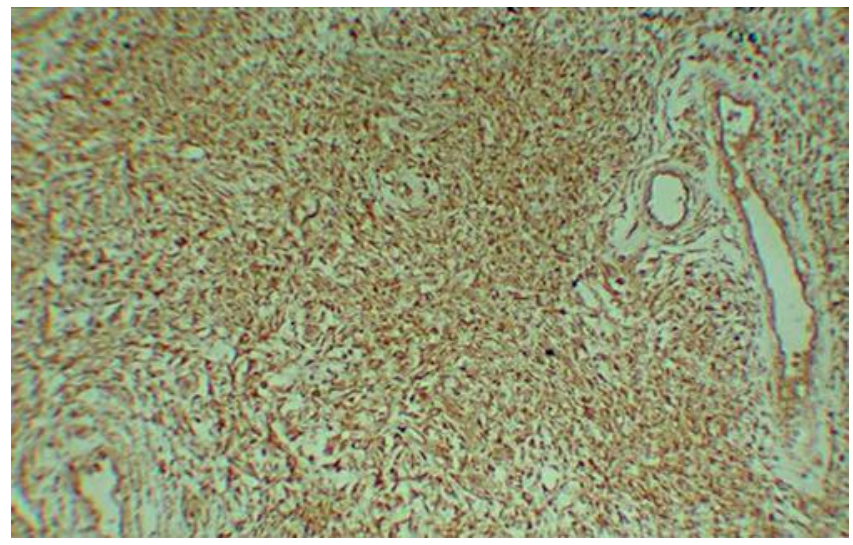

Fig. 6. Diffuse positive staining of the mass with vimentin. Original magnification $\times 10$.

\section{References}

1 De Moulin D: Paul Barbette, M.D.: a seventeenth-century Amsterdam author of best-selling textbooks. Bull Hist Med 1985;59:506-514.

2 Noble I: Master Surgeon: John Hunter. New York, J. Messner, 1971, p 185.

-3 Onkendi EO, Grotz TE, Murray JA, Donohue JH: Adult intussusception in the last 25 years of modern imaging: is surgery still indicated? J Gastrointest Surg 2011;15:1699-1705.

-4 Azar T, Berger DL: Adult intussusception. Ann Surg 1997;226:134-138.

5 Hasegawa T, Yang P, Kagawa N, Hirose T, Sano T: CD34 expression by inflammatory fibroid polyps of the stomach. Mod Pathol 1997;10:349-361.

6 Yakan S, Caliskan C, Makay 0, Denecli AG, Korkut MA: Intussusception in adults: clinical characteristics, diagnosis and operative strategies. World J Gastroenterol 2009;15:1985-1989.

7 Lasithiotakis K, Grisbolaki E, Filis D, Athanasakis I, Zoras O, Chalkiadakis G: Ileocolic intussusception precipitated by diagnostic colonoscopy: a case report. Surg Laparosc Endosc Percutan Tech 2012;22: e161-e163.

$\checkmark 8$ Cera SM: Intestinal intussusception. Clin Colon Rectal Surg 2008;21:106-113.

$>9$ Begos DG, Sandor A, Modlin IM: The diagnosis and management of adult intussusception. Am J Surg 1997;173:88-94.

10 Merine D, Fishman EK, Jones B, Siegelman SS: Enteroenteric intussusception: CT findings in nine patients. AJR Am J Roentgenol 1987;148:1129-1132.

11 Park SB, Ha HK, Kim AY, Lee SS, Kim HJ, Park BJ, Jin YH, Park SH, Kim KW: The diagnostic role of abdominal CT imaging findings in adult intussusception: focused on the vascular compromise. Eur J Radiol 2007;62:406-415.

12 Nagorney DM, Sarr MG, McIlrath DC: Surgical management of intussusception in the adult. Ann Surg 1981;193:230-236.

13 Marinis A, Yiallourou A, Samanides L, Dafnios N, Anastasopoulos G, Vassiliou I, Theodosopoulos T: Intussusception of the bowel in adults: a review. World J Gastroenterol 2009;15:407-411.

-14 Eisen LK, Cunningham JD, Aufses AH Jr: Intussusception in adults: institutional review. J Am Coll Surg 1999;188:390-395.

15 Goh BK, Quah HM, Chow PK, Tan KY, Tay KH, Eu KW: Predictive factors of malignancy in adults with intussusception. World J Surg 2006;30:1300-1304.

16 Vasiliadis K, Kogopoulos E, Katsamakas M, et al: Ileoileal intussusception induced by a gastrointestinal stromal tumor. World J Surg Oncol 2008;6:133. 